\title{
REPRESENTACIONES SOCIALES DE LA CONTAMINACIÓN DEL AIRE Y LAS ESTUFAS DE LEÑA EN DIFERENTES NIVELES SOCIOECONÓMICOS DE LA CIUDAD DE TEMUCO, CHILE
}

\author{
Boris ÁLVAREZ ESCOBAR y Álex BOSO GASPAR*
}

Universidad de La Frontera. Avenida Francisco Salazar 1145, Temuco, Chile, C. P. 4811230

* Autor para correspondencia: alex.boso@ufrontera.cl

(Recibido marzo 2017; aceptado octubre 2017)

Palabras clave: redes semánticas naturales, conciencia, conducta ambiental

\section{RESUMEN}

La contaminación del aire es una problemática urgente a nivel nacional, ya que afecta la salud de millones de personas. Temuco es la tercera ciudad más contaminada de Chile, en la que el uso residencial de estufas de leña es la principal fuente de contaminación atmosférica. Pese a los esfuerzos por revertir esta situación, la reducción de emisiones no ha disminuido como se esperaba. En parte, una posible explicación de este fenómeno radica en las percepciones que tienen los usuarios de las estufas de leña sobre su uso y sobre la problemática de contaminación en Temuco. Este estudio busca describir las representaciones sociales de la contaminación del aire y de las estufas de leña desde la visión de sus usuarios, quienes residen en la ciudad de Temuco. Se empleó un diseño transeccional descriptivo, con la técnica de redes semánticas naturales. Los resultados muestran que los participantes vinculan la contaminación del aire con el humo, las enfermedades y el esmog. Por otra parte, las estufas de leña se asocian al calor, a la calefacción y a la contaminación. Los resultados reflejan una creciente conciencia del vínculo entre contaminación, enfermedad y estufas de leña. Además, estas estufas muestran en sus atributos un carácter multifuncional más allá de entregar calor para el hogar, sino también para cocinar y secar la ropa. Los sectores económicos muestran visiones diferenciadas sobre las estufas de leña y su vínculo con la contaminación del aire.

Key words: natural semantic networks, consciousness, environmental behavior

\begin{abstract}
Air pollution is an urgent problem at national level, as it seriously affects the health of millions of people. Temuco is the third most polluted city in Chile, with the residential use of wood stoves as the main source of atmospheric pollution in this area. Despite the efforts to reverse this situation, the emissions reduction has not decreased as expected. A possible explanation for this phenomenon might be the stakeholders' perceptions about the use of woodstoves and about contamination issues in Temuco. This study aims to describe the social representations of air pollution and woodstoves from the perspective of their users whom reside in Temuco. A transectional descriptive design was employed according to the natural semantic network technique. The results show that participants associate air pollution with smoke, diseases and smog. On the other
\end{abstract}


hand, woodstoves are associated with heat, central heating and pollution. The results reflect a growing awareness of the link between pollution, disease and woodstoves. In addition, woodstoves show in their attributes a multifunctional character beyond heating, cooking and drying of clothes. The economic sectors show different visions about woodstoves and their relationship with air pollution.

\section{INTRODUCCIÓN}

La contaminación del aire constituye una de las principales amenazas para la salud pública a nivel global. El $92 \%$ de la población mundial vive en lugares donde los niveles de calidad del aire superan los límites establecidos, lo que provoca 4.3 y 3.7 millones de muertes al año por contaminación atmosférica en interiores y exteriores, respectivamente (OMS 2016a, OMS 2016b). Los contaminantes del aire nocivos para la salud son diversos y uno de los que ha presentado mayor atención es el material particulado (MP), ya sea grueso $\left(\mathrm{MP}_{10}\right)$ o fino $\left(\mathrm{MP}_{2.5}\right)$. Debido a su diminuto tamaño, este contaminante puede introducirse fácilmente en las vías respiratorias, por lo que las toxinas pueden distribuirse por el cuerpo a través de la sangre y generar graves consecuencias en la salud (Cortés y Ridley 2013). A corto plazo, se pueden observar dificultades para respirar, irritación en los ojos y dolores de cabeza. Por otro lado, en exposiciones prolongadas se puede apreciar el desarrollo de arritmia, ataques de asma, ataques al corazón, enfermedad isquémica del corazón, bronquitis crónica, neumonía (especialmente en menores de 5 años) e incluso cáncer de pulmón (Hine et al. 2007, Rivas et al. 2008, Oyarzún 2010, Carazo et al. 2013, Gascon y Sunyer 2015, INE 2016, Ubillaa y Yohannessen 2017).

En Chile, más de 10 millones de personas se encuentran expuestas a promedios anuales de concentración de $\mathrm{MP}_{2.5}$ por sobre la norma establecida de $20 \mu \mathrm{g} / \mathrm{m}^{3}$. Debido a esto se atribuyen más de 4000 muertes prematuras al año en toda la población nacional (Rivas et al. 2008, SINIA 2010, INE 2015). El $\mathrm{MP}_{2.5}$ es el contaminante que más significativamente se asocia con eventos de mortalidad y morbilidad en Chile (MMA 2011, INE 2015).

Con emisiones anuales de $32 \mu \mathrm{g} / \mathrm{m}^{3}$ de $\mathrm{MP}_{2.5}$, Temuco, una ciudad intermedia del sur de Chile (287 850 hab, INE 2015) es considerada como la tercera ciudad más contaminada del país (MMA 2014). Esta problemática se arrastra desde los años 90, cuando Temuco evidenció mayores índices de contaminación atmosférica que Santiago debido a los altos niveles de $\mathrm{MP}_{10}$ registrados (Oyarzún 2006). Pese a ello, fue a partir de 2005 cuando se declaró como zona saturada de $\mathrm{MP}_{10}$, siendo la segunda ciudad del país en recibir esta clasificación después de Santiago (Armijo et al. 2013, Salini 2014).

En los últimos años las autoridades han implementado una serie de medidas de descontaminación. Sin embargo, a pesar de los esfuerzos el problema sigue vigente en la zona, declarada adicionalmente en 2013 como zona saturada de $\mathrm{MP}_{2.5}$ (Palma y Villarroel 2005, MMA 2016). Por poner un ejemplo, sólo en la primera mitad de 2016, se registraron 70 días con niveles de concentración de $\mathrm{MP}_{10}$ por sobre la norma de $150 \mu \mathrm{g} / \mathrm{m}^{3}$ al día, de los cuales 34 fueron declarados preemergencia ambiental al presentar concentraciones de entre $240-329 \mu \mathrm{g} / \mathrm{m}^{3}$ y 20 días como emergencia ambiental con concentraciones de $330 \mu \mathrm{g} / \mathrm{m}^{3}$ o superiores (MMA 2016).

A nivel nacional, las principales fuentes emisoras de $\mathrm{MP}_{2.5}$ son las fundidoras de cobre, las centrales termoeléctricas y la calefacción residencial de leña (MMA 2011, 2013, INE 2015). No obstante, esta última ha sido referenciada por diversos estudios como el principal factor de la contaminación atmosférica en el sur de Chile, al explicar aproximadamente $80 \%$ del MP presente en el aire de esta área geográfica (Sanhueza et al. 2004, MMA 2011, 2013, Cortés y Ridley 2013, INE 2015). En Temuco, el uso de leña residencial puede llegar a explicar hasta $96 \%$ del $\mathrm{MP}_{2.5}$ (Territorio Mayor 2015).

Tal y como señalan diversas fuentes consultadas, el consumo de leña es la fuente calórica más común en Temuco. Está presente en $80 \%$ de los hogares urbanos y prácticamente en $100 \%$ de los rurales. Sus principales usos son para cocinar y como calefacción, especialmente en el periodo invernal donde las temperaturas disminuyen hasta $\operatorname{los}-5^{\circ} \mathrm{C}$ (MinEnergía 2015). De esta forma, el consumo de leña puede llegar hasta los $500000 \mathrm{~m}^{3} /$ año (Rivas et al. 2008, Cortés y Ridley 2013).

Frente a esta situación, el control de la contaminación atmosférica generado por las estufas de leña se ha convertido en uno de los principales desafíos para las autoridades ambientales, tanto en el ámbito local como en el nacional (Cortés y Ridley 2013, MMA 2013, 2014). Los constantes estados de preemergencia y emergencia ambiental han forzado a 
las autoridades a tomar una serie de medidas (MMA 2011, 2013, 2014), entre las que destacan los planes de descontaminación atmosférica que se implementaron en las comunidades de Temuco y de Padre Las Casas en 2010 con el decreto supremo 78 (Montory 2012). El plan vigente (2014-2018) propone cuatro ámbitos de trabajo para combatir los factores detonantes de la contaminación del aire: i) regulación del uso de leña y mejorar su calidad, ii) mejoramiento o recambio de los artefactos de leña, iii) mejoramiento de las viviendas (reacondicionamiento y búsqueda de viviendas más eficientes) y iv) educación ambiental (Armijo et al. 2013, MMA 2013, 2014).

En esta misma línea, se han establecido los planes de alerta ambiental sanitaria como medidas paliativas de acción a corto plazo para combatir los periodos de alerta, preemergencia y emergencia ambiental. Estos se han enfocado en la regulación de la combustión residencial, por lo que se considera la prohibición de venta y uso de leña húmeda, la difusión de información respecto a los puntos de venta de leña certificada, la restricción del uso de leña durante horarios y zonas específicas y el reemplazo de calefactores por otros menos contaminantes (MMA 2014).

A pesar de los esfuerzos por parte de las autoridades para alcanzar estándares aceptables de la calidad del aire en Temuco, los avances en la reducción del MP no han sido significativos. Durante los últimos inviernos los estados de alerta ambiental siguen siendo recurrentes (MMA 2014, 2016). En este sentido, cabe destacar que pese a la creciente conciencia y preocupación que la población ha mostrado por la problemática de la contaminación atmosférica, aún se mantienen patrones de comportamiento nocivos que contribuyen a su desarrollo. Entre estos, el uso de leña húmeda o no certificada así como el propio uso de las estufas de leña por sobre otras alternativas menos contaminantes (Gifford 2011, Hine et al. 2011).

Diversos autores consideran que para comprender las prácticas ambientales de las personas es necesario dar cuenta de su percepción sobre la contaminación atmosférica, especialmente para el caso del análisis del uso de estufas de leña (Bickerstaff y Walker 2001, Hine et al. 2007, 2011, Gifford 2011, Oltra y Sala 2015, 2016). Una manera de aproximarse a este fenómeno consiste en identificar las representaciones sociales vinculadas a las percepciones. Lo anterior permite estudiar los significados subyacentes a los comportamientos perjudiciales que contribuyen a la contaminación del aire. Según Moscovici (1979), las representaciones sociales constituyen un conocimiento de sentido común compuesto por un sistema de valores, nociones y prácticas, que se construye simbólicamente en el curso de las comunicaciones interindividuales y que proporciona a los individuos formas de comprender, organizar y actuar en la realidad. Estas representaciones poseen un componente intencional, que incluye los objetivos, actitudes y deseos, así como un componente cognitivo, formado por las redes semánticas del individuo-observador (Albala-Bertrand 1996). Ante esto, la técnica de redes semánticas naturales se vuelve pertinente para indagar tanto el conocimiento como la interpretación subjetiva que hacen las personas sobre la contaminación atmosférica y el uso de estufas de leña (Vera et al. 2005).

\section{REVISIÓN DE LA LITERATURA}

Uno de los principales obstáculos reportados en la literatura para sustituir las estufas de leña se encuentra en las limitaciones económicas que pueden presentar sus usuarios. Cuando se comparan otras alternativas de calefacción como la parafina, el gas o los calefactores eléctricos, el funcionamiento de las estufas supone un bajo costo, especialmente si se emplea leña de baja calidad (Hine et al. 2007, Hine et al. 2011, Cortés y Ridley 2013). Sin embargo, la relación entre la tenencia o uso de estufa de leña y el estatus económico no es siempre directa, ya que se deben considerar otros factores para comprender de forma integral la aceptación o rechazo de tecnologías de calefacción alternativas (Hine et al. 2007, 2011, Gifford 2011).

En este sentido, se señala que existe una serie de factores psicológicos que podrían ser relevantes para comprender patrones de comportamiento nocivos vinculados con la contaminación del aire. Esto ayudaría a entender por qué pese a la amplia preocupación y conciencia en las personas, éstas no realizan acciones para mitigar sus efectos sobre la contaminación (Hine et al. 2007, 2011, Gifford 2011, Oltra y Sala 2015, 2016). Siguiendo este planteamiento, los usuarios de estufas de leña tenderían a crear sistemas de justificación que les permitan mantenerse en la comodidad de su statu quo, sin realizar acciones que requieran mayor esfuerzo o implicación para cambiar situaciones complejas (Gifford 2011).

Por otro lado, la resistencia al cambio podría estar mediada por un mecanismo afectivo hacia el fuego o por las sensaciones de bienestar que producen las estufas de leña. Diversos estudios muestran que los usuarios de estufas de leña tienen afectos positivos hacia el calor producido por el fuego de la leña, el cual se tiende a asociar casi de forma innata con sentimientos de comodidad, felicidad y calidez, además de 
sentimientos nostálgicos positivos hacia la niñez y de actividades familiares en torno a la leña (Hine et al. 2007, Reeve et al. 2013). Asimismo, cabe destacar que el uso de estufas de leña es un elemento propio de la identidad y la cultura de la zona sur de Chile, lo que dificulta su sustitución al estar ampliamente ligada con la rutina y prácticas diarias de los usuarios durante los meses de invierno (Shove et al. 2012, Cortés y Ridley 2013, Reeve et al. 2013).

Desde la psicología social se aprecian diversos estudios respecto a la percepción pública de los riesgos a la salud, vinculados con la contaminación atmosférica (Saksensa 2011). Estos se han dirigido fundamentalmente a comprender los factores sociales y culturales que influyen en la percepción, así como identificar las estrategias más efectivas para comunicar estos riesgos (Covello et al. 2001, Renn et al. 2007, Vallejos y Oñate 2013). En este sentido, se señala que la conciencia respecto a los riesgos que implica la contaminación a la salud se ha incrementado en los últimos años. Sin embargo, el vínculo es poco claro respecto a la relación entre ésta y el desarrollo de enfermedades o la presencia de síntomas como dolores de cabeza o garganta, asma, fatiga e irritabilidad. De esta manera, se puede inferir que las personas tampoco establecen un vínculo claro entre las estufas de leña y los riesgos a la salud, ya que la relación es más indirecta (CIEMAT 2014).

El estudio de las representaciones sociales de la contaminación del aire y el uso de estufas de leña que se presenta a continuación pretende contribuir a la comprensión de la base sociocognitiva de las prácticas ambientales cotidianas desplegadas en el espacio público y privado, ofreciendo un recurso importante para intervenir sobre ellas (Gutiérrez 2012, Navarro 2013). En función de los antecedentes presentados, este estudio pretende describir las representaciones sociales de la contaminación del aire y las estufas de leña en los usuarios de diferentes sectores socioeconómicos de la ciudad de Temuco, Chile. Esto se realizará, como se dijo antes, por medio de la técnica de redes semánticas naturales, el cual es un medio útil para acceder empíricamente a la organización cognitiva e interpretación subjetiva que hacen los sujetos sobre el fenómeno de estudio (Castañeda 2016).

Esto se torna relevante, ya que la identificación de la manera en que los sujetos sociales construyen, reconstruyen y transforman la realidad social es un prerrequisito ineludible para lograr una mayor eficacia de las políticas de educación ambiental y transmisión de información (Rico 2016). Además, ofrecen un recurso importante para intervenir sobre ellas (Gutiérrez 2012, Navarro 2013).

\section{MATERIALES Y MÉTODOS}

\section{Participantes}

En este estudio se utilizó un muestro no probabilístico por conveniencia. Como criterios de inclusión se consideraron: (a) ser residente de la ciudad de Temuco, (b) ser mayor de 18 años y (c) ser o haber sido usuario de estufa de leña. Además, se identificó el nivel socioeconómico de los participantes considerando los criterios establecidos por la encuesta ESOMAR (Adimark 2000).

La muestra estuvo compuesta por 90 participantes residentes de la ciudad de Temuco, entre los 18 y 68 años de edad (media $=34.39$, desviación estándar $=$ 11.94 ), de los cuales $34.4 \%$ fueron hombres y $65.6 \%$ mujeres. La totalidad de los participantes fueron alguna vez usuarios de estufas de leña, pero 18 no lo son actualmente. La muestra se dividió de forma equitativa por nivel socioeconómico bajo, medio y alto, con 30 participantes en cada uno.

\section{Instrumentos}

Se empleó la técnica de redes semánticas naturales, la cual a partir de la exposición a palabras estímulo permite una aproximación a la base sociocognitiva de las representaciones sociales de los participantes. De esta forma se posibilita el acceso a la información que tienen en la memoria sobre determinada palabra estímulo, así como el significado que le atribuyen a la misma y la cultura subjetiva que la circunda. Lo anterior juega un papel importante en el desarrollo de sus experiencias y en la manifestación de su conducta (Figueroa et al. 1981, Valdez 2000, Vera et al. 2005).

Para la aplicación de la técnica de redes semánticas naturales, los participantes fueron expuestos a dos conceptos estímulo: "estufas de leña" y "contaminación del aire" (anexo 1). Para cada estímulo, las personas debían escribir un listado de un mínimo de cinco y un máximo de 10 palabras sueltas, que según su perspectiva, mejor definieran dichos conceptos. Luego, debían jerarquizar cada una de estas palabras escritas, otorgándoles un número del 1 al 10 , en función al grado de importancia o cercanía que le atribuyan respecto al concepto estímulo, donde el número más bajo representa mayor importancia y el más alto menor importancia. A partir de esto, se obtiene un conjunto de todas las palabras escritas por los participantes, donde aquellas que más se repiten y que fueron jerarquizadas con mayor importancia, permiten dar cuenta de las representaciones sociales que se construyen en torno a los conceptos estímulo. 
De forma adicional, se incorporó un apartado de preguntas sociodemográficas, considerando edad, género y nivel socioeconómico para describir la muestra y realizar comparaciones con base en esta última característica.

\section{Procedimiento}

Los participantes fueron localizados por medio de contactos clave, es decir, personas residentes de la ciudad de Temuco con capacidad para acceder fácilmente a la muestra poblacional, durante los meses de noviembre y diciembre de 2016. Posteriormente se les notificaron los objetivos de la investigación y se les leyó el consentimiento informado, el cual detalla en qué consiste la participación y los resguardos éticos considerados para la investigación, en el que se resaltó la voluntariedad así como la confidencialidad de la información.

\section{Análisis de datos}

El análisis de los datos se realizó siguiendo el planteamiento original de Figueroa et al. (1981) y posteriormente revisado por otros autores (Valdez 2000, Vera et al. 2005). A partir de los datos se obtuvo: un valor J, referido a la cantidad de palabras diferentes que fueron mencionadas por la totalidad de los participantes (riqueza semántica), el valor $\mathrm{M}$, calculado a partir de la multiplicación entre la frecuencia de cada palabra y el puntaje obtenido a partir de la jerarquía asignada (peso semántico). Además, el análisis semántico del valor $\mathrm{M}$ (conjunto SAM, por sus siglas en inglés), obtenido desde las 10 palabras definidoras que tuvieron mayor valor $\mathrm{M}$ y el valor FMG, que son las puntuaciones del conjunto SAM expresadas en porcentajes tomando como referencia la palabra de mayor puntaje a la cual se le asigna $100 \%$ (distancia semántica).

Posteriormente, en función de los porcentajes obtenidos del valor FMG, se clasificaron las palabras del conjunto SAM de cada concepto estímulo, en las siguientes categorías: (1) núcleo central (100\%), entendido como la palabra más compartida por el grupo y que mejor representa al concepto, (2) atributos esenciales (entre $99 \%$ y $79 \%$ ), correspondientes a un conjunto de palabras sustanciales o imprescindibles para definir el concepto, (3) atributos secundarios (entre $78 \%$ y $58 \%$ ), referidos a palabras que contribuyen a la definición pero de forma accesoria, (4) atributos periféricos (entre $57 \%$ y $37 \%$ ), correspondientes a palabras de menor importancia y que se relacionan indirectamente con el concepto estímulo y (5) atributos personales (36 \% o menor), que por su baja frecuencia no se pueden entender como palabras compartidas por un grupo, sino como nociones particulares de cada persona.

\section{RESULTADOS}

\section{Contaminación del aire}

Respecto al concepto estímulo "contaminación del aire", se obtuvieron 160 palabras diferentes expresadas por los participantes, lo que refleja una amplia riqueza semántica y heterogeneidad del concepto. El cuadro I, muestra el conjunto SAM de este término, con las 10 palabras de mayor peso semántico (valor M) y la distancia semántica de éstas respecto al núcleo central (valor FMG). Del conjunto SAM, se obtiene como núcleo central la palabra "humo" (100\%), siendo ésta la palabra que se compartió con mayor frecuencia entre los participantes para definir la "contaminación del aire". Como atributos esenciales sólo se aprecia la palabra "enfermedad" (96.3\%), la cual también es ampliamente compartida. Posteriormente, se encuentra "esmog" (68.5\%) como atributo secundario, mientras que como atributos periféricos se observan las palabras "industrias" (56.2 \%) y "enfermedades respiratorias" (42\%). Las cinco palabras restantes se consideran atributos personales que detallan aspectos más específicos y menos compartidos respecto a la contaminación, como son "tóxico", "automóviles", "suciedad", "leña mojada" y "mal olor", las cuales reflejan algunas consecuencias de la contaminación, así como posibles causas de la misma.

CUADRO I. ANÁLISIS SEMÁNTICO DEL VALOR M (CONJUNTO SAM) DE "CONTAMINACIÓN DEL AIRE"

\begin{tabular}{lcc}
\hline Palabra & Valor M & Valor FMG (\%) \\
\hline Humo & 302 & 100 \\
Enfermedad & 291 & 96.3 \\
Esmog & 207 & 68.5 \\
Industrias & 170 & 56.2 \\
Enfermedades respiratorias & 127 & 42 \\
Tóxico & 71 & 23.5 \\
Automóviles & 69 & 22.8 \\
Suciedad & 69 & 22.8 \\
Leña mojada & 62 & 20.5 \\
Mal olor & 61 & 20.1 \\
\hline
\end{tabular}

Por otra parte, al observar el conjunto SAM de contaminación del aire según el nivel socioeconómico (Cuadro II), podemos observar que en el nivel bajo, el núcleo central es la palabra "enfermedad" 
CUADRO II. ANÁLISIS SEMÁNTICO DEL VALOR M (CONJUNTO SAM) DE “CONTAMINACIÓN DEL AIRE” SEGÚN EL NIVEL SOCIOECONÓMICO

\begin{tabular}{|c|c|c|c|c|c|c|c|c|}
\hline Bajo & $\begin{array}{c}\text { Valor } \\
\text { M }\end{array}$ & $\begin{array}{c}\text { Valor } \\
\text { FMG (\%) }\end{array}$ & Medio & $\begin{array}{c}\text { Valor } \\
\mathrm{M}\end{array}$ & $\begin{array}{c}\text { Valor } \\
\text { FMG (\%) }\end{array}$ & Alto & $\begin{array}{c}\text { Valor } \\
\mathrm{M}\end{array}$ & $\begin{array}{c}\text { Valor } \\
\text { FMG }(\%)\end{array}$ \\
\hline Enfermedad & 110 & 100 & Esmog & 118 & 100 & Humo & 121 & 100 \\
\hline Humo & 94 & 85.4 & Enfermedad & 89 & 75.4 & Enfermedad & 92 & 76 \\
\hline Industrias/fábricas & 70 & 63.6 & Humo & 87 & 73.7 & Industrias/fábricas & 61 & 50.4 \\
\hline Enf. respiratorias & 41 & 37.2 & Enf. respiratorias & 63 & 53.3 & Esmog & 51 & 42.1 \\
\hline Automóviles & 41 & 37.2 & Mal olor & 45 & 38.1 & Tóxico & 34 & 28 \\
\hline Esmog & 38 & 34.5 & Industrias/fábricas & 39 & 33 & Gases & 33 & 27.2 \\
\hline Leña mojada & 37 & 33.6 & Leña & 31 & 26.2 & Contaminación & 30 & 24.7 \\
\hline Tos & 30 & 27.2 & Vehículos & 31 & 26.2 & Suciedad & 30 & 24.7 \\
\hline Tóxico & 28 & 25.5 & Leña mojada & 25 & 21.1 & $\mathrm{CO}$ & 26 & 21.4 \\
\hline Asma & 27 & 24.5 & Suciedad & 21 & 17.7 & Enf. respiratorias & 23 & 19 \\
\hline
\end{tabular}

(100\%) siendo la más compartida por este grupo. Como atributos esenciales sólo se observa "humo" $(85.4 \%)$, mientras que como atributo secundario se encuentra "industrias" (63.6\%). En los atributos periféricos, están las palabras "enfermedades respiratorias" (37.2\%) y "automóviles" (37.2 \%). Por último, se aprecian cinco conceptos personales, los cuales son "esmog", "leña mojada", "tos", "tóxico" y "asma", que se refieren a consecuencias de la contaminación y a una posible fuente de ésta.

En el caso del nivel socioeconómico medio, el núcleo central es "esmog" (100\%), siendo la más compartida por el grupo. A ésta la siguen, "enfermedad" (75.4\%) y "humo" (73.7 \%) como atributos secundarios, mientras que en los atributos periféricos se aprecian las palabras "enfermedades respiratorias" (53.3 \%) y "mal olor" (38.1\%). Las cinco palabras restantes corresponden a atributos personales: "leña", "vehículos", "leña mojada" y "suciedad". En este grupo no se presentan atributos esenciales.

Para el nivel socioeconómico alto, "humo" (100\%) representa el núcleo central del concepto estímulo "contaminación del aire". Como atributo secundario, se observa la palabra "enfermedad" (76\%) y como atributos periféricos las palabras "industrias" $(50.4 \%)$ y "esmog" (42.1\%). Por último, las seis palabras restantes representan atributos personales como son "tóxico", "gases", "contaminación", "suciedad", "monóxido de carbono" (CO) y "enfermedades respiratorias". En este grupo tampoco se presentan atributos esenciales.

\section{Estufas de leña}

Respecto al concepto estímulo "estufas de leña", se obtuvieron 123 palabras diferentes, lo que también representa una amplia riqueza semántica y heterogeneidad del concepto. En el cuadro III, se muestra el conjunto SAM de esta palabra. El núcleo central obtenido es "calor" (100\%), siendo el término más compartido para definir el estímulo. Como atributos secundarios se observan las palabras "contaminación" (69.7\%), "humo" (63.3\%) y "fuego" (60.6\%), mientras que como atributos periféricos están los conceptos "calefacción" (46 \%) y "leña" (40.2 \%). Las cuatro palabras restantes se consideran atributos personales que mencionan algunas características más específicas del concepto: "hogar", "cocinar", "cenizas" y "leña seca". Cabe destacar que en este caso la palabra estímulo no presenta atributos esenciales, por lo que da un salto desde el núcleo a lo secundario.

CUADRO III. ANÁLISIS SEMÁNTICO DEL VALOR M (CONJUNTO SAM) DE "ESTUFAS DE LEÑA"

\begin{tabular}{lcc}
\hline Palabra & Valor M & Valor FMG (\%) \\
\hline Calor & 447 & 100 \\
Contaminación & 312 & 69.7 \\
Humo & 283 & 63.3 \\
Fuego & 271 & 60.6 \\
Calefacción & 206 & 46 \\
Leña & 180 & 40.2 \\
Hogar & 135 & 30.2 \\
Cocinar & 78 & 17.4 \\
Cenizas & 74 & 16.5 \\
Leña seca & 71 & 15.8 \\
\hline
\end{tabular}

Por otra parte, al revisar el conjunto SAM de estufas de leña según nivel socioeconómico (Cuadro IV) se puede apreciar que en el nivel bajo, el núcleo central es la palabra "calor" (100\%) siendo el término más compartido por este grupo. Posteriormente, 
CUADRO IV. ANÁLISIS SEMÁNTICO DEL VALOR M (CONJUNTO SAM) DE “ESTUFAS DE LEÑA" SEGÚN EL NIVEL SOCIOECONÓMICO

\begin{tabular}{lcccccccc}
\hline Bajo & $\begin{array}{c}\text { Valor } \\
\text { M }\end{array}$ & $\begin{array}{c}\text { Valor } \\
\text { FMG (\%) }\end{array}$ & \multicolumn{1}{c}{ Medio } & $\begin{array}{c}\text { Valor } \\
\text { M }\end{array}$ & $\begin{array}{c}\text { Valor } \\
\text { FMG (\%) }\end{array}$ & Alto & Valor M & $\begin{array}{c}\text { Valor } \\
\text { FMG (\%) }\end{array}$ \\
\hline Calor & 146 & 100 & Calor & 132 & 100 & Calor & 169 & 100 \\
Fuego & 79 & 54.10 & Humo & 118 & 89.3 & Contaminación & 141 & 83.4 \\
Contaminación & 75 & 51.30 & Calefacción & 108 & 81.8 & Fuego & 106 & 62.7 \\
Calefacción & 68 & 46.50 & Contaminación & 96 & 72.7 & Humo & 103 & 60.9 \\
Cocinar & 66 & 45.20 & Fuego & 86 & 65.1 & Hogar & 79 & 46.7 \\
Humo & 62 & 42.40 & Leña & 66 & 50 & Leña & 63 & 37.2 \\
Leña & 51 & 34.90 & Leña seca & 45 & 34 & Invierno & 40 & 23.6 \\
Hogar & 46 & 33.50 & Cenizas & 38 & 28.7 & Acogedor & 38 & 22.4 \\
Secar ropa & 31 & 21.20 & Dinero & 29 & 21.9 & Combustión & 32 & 18.9 \\
Frío & 29 & 19.80 & Estufa & 26 & 19.6 & Madera & 31 & 18.3 \\
\hline
\end{tabular}

se aprecia un salto hacia los atributos secundarios, donde se ubican "fuego", "contaminación", "calefacción", "cocinar" y "humo". Las cuatro palabras restantes "leña", "hogar", "secar ropa" y "frío", se consideran atributos personales. En este grupo no se aprecian atributos esenciales ni atributos periféricos de la palabra estímulo.

Para el nivel socioeconómico medio, el núcleo central también es el "calor" (100\%), siendo la palabra más compartida por el grupo. Los atributos esenciales los componen los conceptos "humo" (89.3\%) y "calefacción" (81.8\%). Les siguen "contaminación" (72.1\%) y "fuego" (65.1\%) como atributos secundarios, mientras que "leña" (50\%) se posiciona como un atributo periférico. Las últimas cuatro palabras "leña seca", "cenizas", "dinero" y "estufa" son mencionadas como atributos personales.

En el caso del nivel socioeconómico alto, al igual que en los dos grupos anteriores, "calor" (100\%) es el núcleo central para definir las estufas de leña. Como atributo esencial se aprecia la palabra "contaminación" (83.4\%). Le siguen "fuego" (62.7\%) y "humo" $(60.9 \%)$ como atributos secundarios y "hogar" (46.7 \%) y "leña" (37.2\%) como atributos periféricos. Por último, se ubican las palabras "invierno", "acogedor", "combustión" y "madera" como atributos personales.

\section{CONCLUSIONES Y DISCUSIÓN}

En función de los resultados, se observa que para los usuarios de estufas de leña impera una representación de la contaminación del aire en torno a características visibles como el humo y el esmog, los cuales son fácilmente perceptibles especialmente durante episodios de contaminación críticos (MMA 2016).
Asimismo, asocian estrechamente la contaminación con "enfermedades". Esto concuerda con estudios previos donde se ha observado una creciente conciencia respecto a las consecuencias de la contaminación en la población (Hine et al. 2011, CIEMAT 2014).

La predominante mención de la palabra "industrias", como una fuente de la contaminación atmosférica no es una percepción concordante con la realidad de la zona (MMA 2015). Asimismo, la menor referencia del uso de leña mojada como causa posible de la contaminación del aire podría indicar una escasa consideración de las estufas de leña como fuente principal de contaminación en la zona, al ser mencionada con la misma o menor frecuencia que otras fuentes que han tenido un menor impacto (MMA 2015). Hasta cierto punto este hecho es relativamente paradójico, pues en los últimos años, las autoridades locales han desarrollado numerosas campañas de provisión de información y comunicación del riesgo. A pesar de ello, los datos apuntan a que la percepción de los individuos sobre la contaminación del aire y sus fuentes, no se corresponden del todo con la realidad de su entorno. En cierto sentido, este hallazgo pone en tela de juicio estudios previos en los que se ha afirmado que la mayoría de las personas conscientes de la calidad del aire residen en hogares cercanos a alguna fuente directa de contaminación (Bickerstaff y Walker 2001, Saksena 2011, Simone et al. 2012).

Con relación a las estufas de leña, prevalece la representación social en torno al "calor", lo que refleja la función y beneficio fundamental que aportan, siendo esta una característica acorde con las bajas temperaturas que presenta Temuco, especialmente en los periodos de invierno (Rivas et al. 2008, Cortés y Ridley 2013). En segundo lugar, se aprecia una concepción de las estufas de leña ligada con las 
consecuencias adversas de su uso para el ambiente, es decir, las estufas son consideradas como una fuente que contribuye a esta problemática por el "humo" que emanan (Hine et al. 2011). Al revisar los atributos personales, se aprecia una noción de las estufas de leña en torno a una sensación hogareña o de estar en casa y se destaca su carácter multifuncional, vinculado especialmente con funciones de "cocinar" y "secar ropa", no limitado exclusivamente a generar calor.

Por otro lado, no se observaron palabras de afectos positivos en el conjunto SAM de las estufas de leña como se podía esperar desde lo propuesto por otras investigaciones (Hine et al. 2007, Reeve et al. 2013). Sin embargo, a un nivel más específico sí emergieron una amplia diversidad de palabras vinculadas a estas emociones como son "amor", "confortable", "cálido", "tranquilidad", "agradable", "alivio" y "ambiente familiar", así como aspectos relativos a una faceta social como "familia", "amigos" y "unidad". En este sentido, queda pendiente observar los resultados bajo análisis en categorías de segundo orden que permitan la agrupación de palabras en conceptos más abstractos e inclusivos y ver cómo evolucionan los resultados con una muestra más amplia.

Pese a la gravedad de la contaminación del aire en Temuco debido al extendido uso de estufas de leña, la palabra "contaminación" aparece como un concepto secundario de las representaciones sociales de las estufas. Tanto así que sólo para los participantes que residen en el sector alto de la ciudad, la contaminación es un atributo esencial asociado estrechamente con las estufas de leña. Por otro lado, los participantes de los demás niveles socioeconómicos percibieron un vínculo más débil, siendo secundario en los del sector medio y periférico para el nivel bajo. Este hallazgo es relevante para los estudios de percepción de contaminación atmosférica y comunicación del riesgo a la salud, por lo que debe tenerse en consideración dadas sus posibles implicaciones prácticas. En este sentido, cabe destacar que, en la gestión de la calidad del aire, informar y facilitar las conductas individuales de involucramiento y la reducción de la contaminación atmosférica suelen ser objetivos transversales en la población (Oltra et al. 2017). Sin embargo, los resultados de este artículo indican que las acciones de comunicación del riesgo y las campañas educativas deberían ser sensibles a un público diferenciado.

Tal y como se plantea al inicio del artículo, existe un reducido cuerpo de investigaciones que ha abordado la percepción del riesgo a la salud de personas residentes en ciudades contaminadas por el uso de las estufas de leña, menos aún atendiendo a la variación que se puede dar por estrato socioeconómico (Hine et al. 2007, Reeve et al. 2013, Vallejos y Oñate 2013). Algunos estudios realizados en ciudades saturadas por otras fuentes de contaminación del aire concluyeron que la percepción del riesgo a la salud no está asociada con los factores socioeconómicos de los individuos entrevistados (Byrd et al. 1997, 2001). No obstante, los resultados de la presente investigación muestran que en Temuco las personas de estratos socioeconómicos bajos tienen más dificultades para relacionar a las estufas de leña con la contaminación del aire. Aparentemente, esta diferencia podría estar basada en el valor adicional que la multifuncionalidad confiere a los artefactos de leña en las clases sociales más vulnerables. En dichos hogares, los artefactos de leña son un medio más económico de calefacción que las principales tecnologías alternativas (las estufas de combustible o de parafina). Además, por sus características técnicas pueden ser utilizadas en distintas prácticas hogareñas. Las estufas están integradas en las actividades cotidianas del hogar y como indican algunos estudios previos, son un reflejo de su papel en las tradiciones propias de la cultura de los usuarios (Cortés y Ridley 2013). Por consiguiente, las intervenciones a implementar en este ámbito deben ser consideradas cuidadosamente.

En función de lo anterior, resulta necesario que la aplicación de los planes de descontaminación ambiental se fundamenten en medidas estructurales y regulativas con una mirada integral, atendiendo no sólo a los déficits informativos de la población sino también a sus condiciones de vida, necesidades colectivas y prácticas sociales. En este sentido, las políticas de mejoramiento del aislamiento térmico de las viviendas, el apoyo para el recambio de estufas y el desarrollo de intervenciones comunitarias para la autogestión y monitoreo ciudadano de la calidad del aire resultan ser elementos fundamentales en la transición energética de las ciudades intermedias del sur de Chile.

Este estudio presenta algunas limitaciones. Los resultados señalan que las variables psicosociales son un elemento clave para entender los mecanismos de bloqueo y de resistencia al cambio tecnológico que caracterizan el problema de la contaminación atmosférica en las ciudades intermedias del sur de Chile. El análisis de los datos muestra que las nociones de contaminación del aire y estufas de leña evocan una amplia riqueza semántica entre los participantes, aspecto que debería considerarse en los planes de acción. Sin embargo, es evidente que existen otros factores que tienen que ver con las prácticas culturales por un lado, y con factores institucionales por el otro, que son indispensables para comprender 
las dificultades que entraña la transición hacia un sistema energético más sostenible en Temuco y en Padre Las Casas. Por otro lado, resulta difícil discernir el peso que cada uno de estos elementos psicológicos, culturales o institucionales tiene en las barreras que dificultan la transición energética del sistema. En este sentido, algunas de las conclusiones de este estudio deberán ser analizadas en profundidad y puestas a prueba como hipótesis de trabajo en futuras investigaciones.

\section{AGRADECIMIENTOS}

Los autores agradecen el apoyo de la Dra. Marianela Denegri Coria por los comentarios realizados a los informes que precedieron la redacción del artículo. Este documento es producto del proyecto Fondecyt Iniciación 11150262, financiado por el Fondo Nacional de Ciencia y Tecnología (FONDECYT) del Gobierno de Chile. Ambos investigadores, Boris Álvarez y Àlex Boso, declaran ser autores referentes de este artículo.

\section{REFERENCIAS}

Adimark (2000). El nivel socio económico Esomar. Adimark. Manual de aplicación. Santiago, Chile, 8 pp.

Albala-Bertrand L. (1996) For a sociogenetic constructivist didactics of citizenship. Prospects 26 (4), 705-46.

Armijo G., Whitman C. y Roubelat L. (2013). Reacondicionamiento térmico en Temuco-Padre Las Casas, Chile: una ciudad declarada saturada por contaminación aérea. Memorias. XII Encontro Nacional de Conforto no Ambiente Construido ENCAC y VIII Encontro Latinoamericano de Conforto no Ambiente Construido ELACAC. Brasilia. 25 al 27 de septiembre, 1-10 pp.

Bickerstaff K. y Walker G. (2001). Public understandings of air pollution: the 'localisation' of environmental risk. Glob. Environ. Chang. 11 (2), 133-145.

DOI: 10.1016/S0959-3780(00)00063-7

Byrd T. L., VanDerslice J. y Peterson S. K. (1997). Variation in environmental risk perceptions and information sources among three communities in El Paso. Risk 8 (4), 355-372.

Byrd T. L., VanDerslice J. y Peterson S. K. (2001). Attitudes and beliefs about environmental hazards in three diverse communities in Texas on the border with Mexico. Rev. Panam. Salud Públ. 9 (3), 154-160. DOI: $10.1590 / \mathrm{S} 1020-49892001000300004$

Carazo L., Fernández R., González F. y Rodríguez F. (2013). Contaminación del aire interior y su impacto en la patología respiratoria. Arch. Bronconeumol. 49 (1), 22-27. DOI: 10.1016/j.arbres.2012.04.005

Castañeda A. (2016). Las redes semánticas naturales como estrategia metodológica para conocer las representaciones sociales acerca de la investigación en el contexto de la formación profesional de los comunicadores. ESCC 22 (43), 123-168.

CIEMAT (2014). A review of the social research on public perception and engagement practices in urban air pollution. Reporte. Centro de Investigaciones Energéticas, Medioambientales y Tecnológicas. Madrid, España, $54 \mathrm{pp}$.

Cortés A. y Ridley I. (2013). Efectos de la combustión a la leña en la calidad del aire intradomiciliario. La ciudad de Temuco como caso de estudio. Revista INVI 28 (78), 257-271. DOI: $10.4067 / \mathrm{S} 0718-83582013000200008$

Covello V., Peters R., Wojtecki J. y Hyde R. (2001). Risk communication, the west nile virus epidemic, and bioterrorism: responding to the communication challenges posed by the international or unintentional release of a pathogen in an urban setting. J. Urban Health. 78 (2), 382-391.

DOI: $10.1093 /$ jurban/78.2.382

Figueroa J. G., González E. G. y Solís V. M. (1981). Una aproximación al problema del significado: las redes semánticas. RLP 13 (3), 447-458.

Gascon M. y Sunyer J. (2015).Contaminación del aire y salud respiratoria en niños. Arch. Bronconeumol. 51 (8), 371-372. DOI: 10.1016/j.arbres.2015.03.001

Gifford R. (2011). The dragons of inaction: psychological barriers that limit climate change mitigation and adaptation. Am. Psychol. 66 (4), 290-302.

DOI: $10.1037 / \mathrm{a} 0023566$

Gómez A. (2005). El consumo de leña en el sur de Chile ¿por qué nos debe preocupar y qué se puede hacer? Revista Ambiente y Desarrollo 21 (3), 43-47.

Gutiérrez S. (2012). Terrón Amigón, Esperanza, 2010, Educación ambiental. Representaciones sociales y sus implicaciones educativas. Cultura y representaciones sociales 6 (12), 262-269.

Hine D. W., Bhullar N., Marks A., Kelly P. y Scott J. (2011). Comparing the effectiveness of education and technology in reducing wood smoke pollution: a field experiment. J. Environ. Psychol. 31 (4), 282-288. DOI: $10.1016 /$ j.jenvp.2011.05.003

Hine D. W., Marks A. D., Nachreiner M., Gifford R. y Heath Y. (2007). Keeping the home fires burning: the affect heuristic and wood smoke pollution. J. Environ. Psychol. 27 (1), 26-32.

DOI: $10.1016 /$ j.jenvp.2007.01.001

INE (2015). Compendio estadístico región de La Araucanía. Informe anual. Instituto Nacional de Estadísticas. Temuco, Chile, 92 pp. 
INE (2016). Medio ambiente. Informe anual 2015. Instituto Nacional de Estadísticas. Santiago, Chile, 201 pp.

MinEnergía (2015). Medición del consumo nacional de leña y otros combustibles sólidos derivados de la madera. Informe. Ministerio de Energía. Santiago, Chile, 99 pp.

MMA (2011). Informe del estado del medio ambiente. Resumen ejecutivo. Ministerio del Medio Ambiente. Santiago, Chile, 44 pp.

MMA (2013). Primer reporte del estado del medio ambiente. Reporte. Ministerio del Medio Ambiente. Santiago, Chile, 152 pp.

MMA (2014). Planes descontaminación atmosférica: estrategia 2014-2018. Informe. Ministerio del Medio Ambiente. Santiago, Chile, 12 pp.

MMA (2016). Estado de calidad del aire por regiones del país y zonas de interés para alertas sanitarias. Seguimiento norma MP2.5, decreto $\mathrm{n}^{\circ} 12$ del MMA. Resumen Calidad del Aire. Ministerio del Medio Ambiente. Santiago, Chile, 10 pp.

Montory C. H. (2012). Eficacia de los instrumentos del plan de descontaminación de Temuco y Padre Las Casas. Tesis de Licenciatura. Facultad de Ciencias Jurídicas y Sociales. Universidad Austral de Chile. Valdivia, Chile, 48 pp.

Moscovici S. (1979). El psicoanálisis, su imagen y su público. Editorial Huemul. Buenos Aires, Argentina, $363 \mathrm{pp}$.

Navarro O. (2013). Representación social del medio ambiente y de la contaminación del aire: efecto de imbricación de dos objetos. Revista CES Psicología 6 (1), 104-121.

Oltra C. y Sala R. (2015). Communicating the risks of urban air pollution to the public. A study of urban air pollution information services. Rev. Int. Contam. Ambie. 31 (4), 36-375.

Oltra C. y Sala R. (2016). Perception of risk from air pollution and reported behaviors: a cross-sectional survey study in four cities. J. Risk Res. 1-16.

DOI: $10.1080 / 13669877.2016 .1264446$

Oltra C., Sala R., Boso À. y Asensio S. L. (2017). Public engagement on urban air pollution: an exploratory study of two interventions. Environ. Monit. Assess. 189 (6), 296. DOI: 10.1007/s10661-017-6011-6

OMS (2016a). Actualización sobre la $69^{\mathrm{a}}$ asamblea mundial de la salud. Organización Mundial de la Salud [en línea]. http://www.who.int/mediacentre/news/ releases/2016/wha69-27-may-2016/es/ 03/01/2017

OMS (2016b). La OMS publica estimaciones nacionales sobre la exposición a la contaminación del aire y sus repercusiones para la salud. Organización Mundial de la Salud [en línea]. http://www.who.int/mediacentre/news/ releases/2016/air-pollution-estimates/es/ 03/01/2017
Oyarzún M. (2006). Desencuentros entre los conocimientos y las políticas públicas para afrontar la contaminación atmosférica. Rev. Chil. Enf. Respir. 22 (3), 151-154. DOI: $10.4067 / \mathrm{S} 0717-73482006000300001$

Oyarzún M. (2010). Contaminación aérea y sus efectos en la salud. Rev. Chil. Enf. Respir. 26 (1), 16-25. DOI: $10.4067 / \mathrm{S} 0717-73482010000100004$

Palma P. y Villarroel M. (2005). Propuestas ciudadanas para la educación ambiental a partir del problema de contaminación atmosférica en la ciudad de Temuco. Memorias. V Jornadas Interuniversitarias de Investigación. Santiago, 2005. 1-18.

Reeve I., Scott J., Hine D. W. y Bhullar N. (2013). “This is not a burning issue for me": how citizens justify their use of wood heaters in a city with a severe air pollution problem. Energy Policy 57, 204-211. DOI: $10.1016 /$ j.enpol.2013.01.042

Renn O., Carius R., Kastenholz H. y Schulze M. (2007). ERiK - Development of a multi-stage risk communication process. BfR Wissenschaft. Berlin, Alemania, $102 \mathrm{pp}$.

Rico A. (2016). Representaciones sociales de un grupo de estudiantes universitarios colombianos sobre el ambiente. Educ. Pesqui. 42 (4), 1001-1014.

DOI: $10.1590 / \mathrm{s} 1517-9702201603143940$

Rivas E., Barrios S., Dorner A. y Osorio X. (2008). Fuentes de contaminación intradomiciliaria y enfermedad respiratoria en jardines infantiles y salas cunas de Temuco y Padre Las Casas, Chile. Rev. Méd. Chile. 136 (6), 767-774. DOI: 10.4067/S0034-98872008000600013

Territorio Mayor (2015). Estudio actualización diagnóstico territorial para modificación al plan regulador. Informe. Temuco, Chile, 66 pp.

Salini G. A. (2014). Estudio acerca del material particulado emitido en ciudades de tamaño medio al sur de Santiago de Chile. Revista INGE CUC 10 (1), 97-108.

Sanhueza P., Vargas C. y Mellado P. (2004). Impacto de la contaminación del aire por $\mathrm{PM}_{10}$ sobre la mortalidad diaria en Temuco. Rev. Méd. Chile. 134 (6), 754-761. DOI: $10.4067 / \mathrm{S} 0034-98872006000600012$

Saksena S. (2011). Public perceptions of urban air pollution risks. Risk, Hazards and Crisis in Public Policy 2 (1), 1-19. DOI: 10.2202/1944-4079.1075

Simone D., Eyles J., Newbold K. B., Kitchen P. y Williams A. (2012). Air quality in Hamilton: who is concerned? perceptions from three neighbourhoods. Soc. Indic. Res. 108 (2), 239-255.

DOI: $10.1007 / \mathrm{s} 11205-012-0064-2$

Shove E., Pantzar M. y Watson M. (2012). The dynamics of social practice: everyday life and how it changes. 6a ed. SAGE. Nueva Delhi, India, 208 pp.

DOI: $10.4135 / 9781446250655$ 
SINIA (2010). Análisis general del impacto económico y social de la norma de calidad primaria de material particulado 2,5. Expediente. Sistema Nacional de Información Ambiental. Santiago, Chile, 69 pp.

Ubilla C. y Yohannessen K. (2017). Contaminación atmosférica efectos en la salud respiratoria en el niño. Rev. Med. Clin. Condes. 28 (1), 111-118.

DOI: $10.1016 /$ j.rmclc.2016.12.003

Vallejos A. y Oñate M. (2013). Comunicación de riesgos ecológicos: el caso de la contaminación atmosférica en dos ciudades intermedias del sur de Chile. Rev. Int. de Contam. Ambie. 29 (1), 59-75.
Valdez J. L. (2000). Las redes semánticas naturales, usos y aplicaciones en psicología social. 3a ed. Universidad Autónoma del Estado de México. México D.F., 13 pp.

Vera J. A., Pimentel C. E. y Batista F. J. (2005). Redes semánticas: aspectos teóricos, técnicos, metodológicos y analíticos. Ra Ximhai 1 (3), 439-451. 


\section{ANEXO 1}

\section{Cuestionario redes semánticas naturales} I. REDES SEMÁNTICAS NATURALES

a) Escriba un mínimo de 5 y un máximo 10 palabras que a su parecer definan el concepto estímulo"ESTUFAS DE LEÑA". Evite utilizar artículos, preposiciones u otro tipo de partícula gramatical, es decir, intente escribir sólo palabras sueltas (sustantivos, adjetivos y verbos).

\begin{tabular}{|l|l|}
\hline Palabras para "estufas de leña" & Jerarquización \\
\hline & \\
\hline & \\
\hline & \\
\hline & \\
\hline & \\
\hline & \\
\hline & \\
\hline & \\
\hline & \\
\hline
\end{tabular}

Ahora, ya escritas sus palabras, ordénelas jerárquicamente en la columna "jerarquización", donde 1 es la de mayor importancia y cercanía, y 10 es la de menor importancia y cercanía. Es decir, para el concepto estímulo que para usted es más representativa y cercana a la palabra "ESTUFAS DE LEÑA" ingrese el valor 1. Para la segunda más representativa ingrese el valor 2 y así sucesivamente hasta terminar con las palabras que haya ingresado.

b) Escriba un mínimo de 5 y un máximo 10 palabras que a su parecer definan la palabra "CONTAMINACIÓN DEL AIRE". Evite utilizar artículos, preposiciones u otro tipo de partícula gramatical, es decir, intente escribir sólo palabras sueltas (sustantivos, adjetivos y verbos). 
b) Escriba un mínimo de 5 y un máximo 10 palabras que a su parecer definan el concepto "CONTAMINACIÓN DEL AIRE". Evite utilizar artículos, preposiciones u otro tipo de partícula gramatical, es decir, intente escribir sólo palabras sueltas (sustantivos, adjetivos y verbos).

\begin{tabular}{|l|l|}
\hline Palabras para “contaminación del aire” & Jerarquización \\
\hline & \\
\hline & \\
\hline & \\
\hline & \\
\hline & \\
\hline & \\
\hline & \\
\hline & \\
\hline
\end{tabular}

Ahora, ya escritas sus palabras, ordénelas jerárquicamente en la columna "jerarquización", donde 1 es la de mayor importancia y cercanía, y 10 es la de menor importancia y cercanía. Es decir, para la palabra que para usted es más representativa y cercana al concepto "CONTAMINACIÓN DEL AIRE" ingrese el valor 1. Para la segunda más representativa ingrese el valor 2 y así sucesivamente hasta terminar con las palabras que haya ingresado.

\section{DATOS PERSONALES}

1. Edad:

2. Género:

\begin{tabular}{|l|l|}
\hline & Hombre \\
\hline & Mujer \\
\hline
\end{tabular}

3. ¿Actualmente es usuario de estufas de leña?

\begin{tabular}{|l|l|}
\hline & Sí \\
\hline & No \\
\hline
\end{tabular}

4. ¿Alguna vez ha sido usuario de estufas de leña?

\begin{tabular}{|l|l|}
\hline & Sí \\
\hline & No \\
\hline
\end{tabular}


5. ¿Cuál es el nivel de educación que alcanzó la persona que aporta el ingreso principal de este hogar?

\begin{tabular}{|l|l|}
\hline & Educación básica o inferior \\
\hline & Básica completa \\
\hline & Media incompleta (incluyendo media técnica) \\
\hline & Media completa. Técnica incompleta \\
\hline & Universitaria incompleta. Técnica completa \\
\hline & Universitaria completa \\
\hline & Posgrado (maestría, doctorado o equivalente) \\
\hline
\end{tabular}

6. ¿Cuál es la profesión o trabajo de la persona que aporta el principal ingreso de este hogar? Por favor describa

\begin{tabular}{|l|l|}
\hline & $\begin{array}{l}\text { Trabajos menores ocasionales e informales (lavado, aseo, servicio doméstico ocasional, } \\
\text { cuidador de autos, limosna) }\end{array}$ \\
\hline Oficio menor, obrero no calificado, jornalero, servicio doméstico con contrato \\
\hline $\begin{array}{l}\text { Obrero calificado, capataz, junior, micro empresario (kiosco, taxi, comercio menor, } \\
\text { ambulante) }\end{array}$ \\
\hline $\begin{array}{l}\text { Empleado administrativo medio y bajo, vendedor, secretaria, jefe de sección. Técnico } \\
\text { especializado. Profesional independiente de carreras técnicas (contador, analista de sistemas, } \\
\text { diseñador, músico). Profesor de primaria o secundaria }\end{array}$ \\
\hline $\begin{array}{l}\text { Ejecutivo medio (gerente, sub-gerente), gerente general de empresa media o pequeña. } \\
\text { Profesional independiente de carreras tradicionales (abogado, médico, arquitecto, ingeniero, } \\
\text { agrónomo) }\end{array}$ \\
$\begin{array}{l}\text { Alto ejecutivo (gerente general) de empresa grande. Directores de grandes empresas. } \\
\text { Empresarios propietarios de empresas medianas y grandes. Profesionales independientes } \\
\text { de gran prestigio }\end{array}$ \\
\hline
\end{tabular}

